\title{
On k-spanned projective surfaces
}

\author{
Edoardo Ballico \\ Dip. di Matematica, Università di Trento, 38050 Povo (TN),Italy
}

This note can be considered as an appendix to [BS], since here we give an improvement of [BS], th.2.4.

First we recall a few notations. We work over the complex number field. Let $T \subset P^{N}$ be a scheme of dimension 0; $T$ is called curvilinear if it is contained in a smooth curve, or equivalently if it has embedding dimension at most 1 . Let $\mathrm{X}$ be a complete variety embedded in a projective space by a linear subspace $\mathrm{W}$ of $\mathrm{H}^{0}(\mathrm{~S}, \mathrm{~L}), \mathrm{L} \in \mathrm{Pic}(\mathrm{X}) .(\mathrm{X}, \mathrm{W})$ (or $\mathrm{X}$ if there is no danger of misunderstanding) is called $\mathrm{k}$-spanned if for all curvilinear subschemes $T$ of $X$ with length $(T)=k+1$, the restriction map from $W$ to $H^{0}(T, L T)$ is surjective. $L$ is called $k$-spanned if $\left(X, H^{0}(X, L)\right)$ is $k$-spanned.

Here we prove the following result.

Theorem Let $(S, W)$ be a $k$-spanned smooth surface with $k \geq 3$. Then $\operatorname{dim}(W) \geq k+5$.

Proof. Set $\mathbf{P}:=\mathbf{P}(\mathrm{W})$, hence $\mathrm{S} \subset \mathbf{P}$. Assume $\mathbf{w}:=\operatorname{dim}(\mathrm{W}) \leq \mathrm{k}+4$. Take a general hyperplane $\mathrm{H}$ of $\mathbf{P}$ and set $\mathrm{C}:=\mathrm{H} \cap \mathrm{S}$. Then $\mathrm{C}$ is a smooth, $\mathrm{k}$-spanned curve in $\mathrm{H}$. It is easy to check that the projection from a point of a smooth $m$-spanned curve, $m \geq 2$, is a smooth ( $m-1)$-spanned curve in the appropriate projective space.

After (k-2) general projections, we find a smooth 2-spanned curve $Z$ in a projective space $U$, $\operatorname{dim}(U) \leq 4$. If $\operatorname{dim}(U)<4$, this is a contradiction. Assume $\operatorname{dim}(U)=4$, hence $w=k+4$. Let $d, g$ be respectively the degree and genus of $Z$. Note that $S$ has sectional genus g. By Castelnuovo's formula for the number of trisecant lines to a smooth curve in $P^{4}$ ([LB],p.182) we get $d \leq 8, g \leq 5$, and that $Z$ is linearly normal. But then $C$ and $S$ are linearly normal. In [BS],5.1,5.2,5.3, there is a complete classification of all linearly normal surfaces with $k \geq 2$ and sectional genus $g \leq 5$. For the surfaces listed in [BS], $\$ 5$, with $k \geq 3$, we have always $h^{0}(S, L) \geq k+5$, proving the theorem.

This note was bom in the warm atmosphere of Max-Planck-Institut (Bonn).

\section{References}

[BS] M. Beltrametti, A.J. Sommese: On $k$-spannedness for projective surfaces, preprint MPI/88 - 14.

[LB] P. Le Barz: Formule multi-secantes pour les courbes gauches quelconques, in Enumerative geometry and classical algebraic geometry, p. 165-197, Progress in Math. 24, Birkauser. 